\title{
A new approach to produce polystyrene monoliths by gelation and capillary shrinkage
}

\author{
Dewang $\mathrm{Li}^{1}$, Yaqian Deng ${ }^{2}$, Jingyi Xia ${ }^{1}$, Zhitan $\mathrm{Wu}^{1}$, Tongxin Shang ${ }^{1}$, Pei $\mathrm{Li}^{1}$, Junwei Han ${ }^{1}$, \\ Ying Tao ${ }^{1^{*}}$ and Quan-Hong Yang ${ }^{1,3}$
}

\begin{abstract}
Polymeric monoliths are of great interest in a variety of applications. A new gelation approach to produce a mechanically stable polystyrene (PS) gel directly from its microemulsion is reported. To produce a PS gel, the as-prepared microemulsion is first demulsified by adding selected watermiscible organic solvents. The small PS latex particles liberated from the surfactant are assembled into a piece of bulk material at an appropriate temperature with a high degree of entanglement of the polymer chains. It is found that the $d^{2} T / \eta$ value is an important parameter to evaluate the gelation ability of the organic solvents and helps determine the gelation conditions. Finally, PS monoliths are obtained by capillary drying and their pore structures can be effectively tuned by changing the gelation time and the amount of solvent exchanged with water. This allows the controlled preparation of bulk PS artefacts with densities in the range of 0.06 to $1.14 \mathrm{~g} \mathrm{~cm}^{-3}$. This simple method of PS monolith production avoids the use of shaping tools or chemical templates, needs less energy, and is a promising alternative approach to design either integrated porous or compact polymer materials.
\end{abstract}

Keywords: polystyrene, monolith, demulsification, gelation, capillary shrinkage

\section{INTRODUCTION}

A bulk material rather than a powder is preferred for many material applications especially when used as a support or matrix, since it takes full advantage of the mechanical stability and easy reuse [1]. Polystyrene (PS) is a common polymer and has been widely used in many fields, such as plastic products, insulation materials, catalyst supports, chromatographic separation, gas or oil absorption [2-8]. Like many thermoplastics, extrusion, compression or injection molding is usually used for shaping PS in industry, but many attempts have been made to produce a functional polymeric monolith without complex shaping equipment and high energy consumption.

One of the most commonly used methods to produce a porous PS monolith is high internal phase emulsion (HIPE) polymerization [9], which relies on the selection of a surfactant or solid particle to stabilize the emulsion where a large amount of the volume, more than 74 vol.\% of the dispersed aqueous phase (i.e. internal phase), is encapsulated by a small amount of the continuous monomer phase $[10,11]$. Bulk PS is then obtained by the copolymerization of the styrene (St) monomer and a crosslinking agent in the continuous phase with the internal phase acting as a template. Since the pioneering work by Morbidelli's group [12,13], reactive gelation has been extensively used to prepare various macroporous polymeric monoliths with a highly crosslinked structure. This method features two important processes, pore formation by the use of porogens and post-polymerization of the residual monomer. An alternative route, phase separation induced by cooling or solvent evaporation has also been widely used to fabricate polymer monoliths $[14,15]$. Macroporous PS foams can also be fabricated by the UV-initiated polymerization of foamed emulsions with the help of photoinitiators [16]. Most of these methods are highly dependent on the assistance of a mold, templates, porogens, toxic solvents or complicated crosslinking and stabilizing agents to produce polymeric monoliths with the desired structure for different appli-

\footnotetext{
${ }^{1}$ Nanoyang Group, State Key Laboratory of Chemical Engineering, School of Chemical Engineering and Technology, Tianjin University, Tianjin 300350, China

${ }^{2}$ Shenzhen Key Laboratory for Graphene-Based Materials, Engineering Laboratory for Functionalized Carbon Materials, Graduate School at Shenzhen, Tsinghua University, Shenzhen 518055, China

${ }^{3}$ Joint School of National University of Singapore and Tianjin University, International Campus of Tianjin University, Fuzhou 350207, China

* Corresponding author (email: yingtao@tju.edu.cn)
} 
cations. Therefore, more effort needs to be made in exploring alternative methods for PS monolith production with less reliance on the preparation conditions and more choice in tailoring its microstructure.

Here, we report a new PS gelation process which is effective in preparing a monolith starting from its oil/ water microemulsion. PS gelation occurs upon the addition of a water-miscible organic solvent, which acts as an effective demulsifier of the microemulsion. Rapid gelation in only a few minutes at ambient temperature was observed with acetone as the demulsification solvent (Video S1). Note that heating is necessary for most cases. Moreover, densification of the as-prepared gels was successfully achieved, indicating the possibility of tailoring the pore structure of the PS monolith and preparing porous or compact polymer bulk materials by capillary shrinkage, a method previously used for graphene-based hydrogels $[17,18]$.

\section{EXPERIMENTAL SECTION}

\section{Emulsion polymerization}

The St monomer (AR, Aladdin) was purified by washing in $1 \mathrm{~mol} \mathrm{~L}^{-1} \mathrm{NaOH}$ solution to get rid of trace polymerization inhibitors. As listed in Table S1, weighed amounts of St and sodium dodecyl sulfate (SDS) (AR, Aladdin) were added to $100 \mathrm{~mL}$ deionized (DI) water in a 250-mL four-neck flask under stirring, then $0.01 \mathrm{~g} \mathrm{~mL}^{-1}$ $\mathrm{K}_{2} \mathrm{~S}_{2} \mathrm{O}_{8}$ (99.5\%, Real \& Lead) aqueous solution was added. After purging with Ar for 15 min under stirring to remove the remaining oxygen, the mixture was placed in a $70^{\circ} \mathrm{C}$ water bath where it was kept for $6 \mathrm{~h}$. As it reached the target temperature, the transparent solution gradually turned a translucent blue color for microemulsions or an opaque white color for emulsions with larger PS latex sizes.

\section{Preparation of the PS monolith}

Typically, $10 \mathrm{~mL}$ water-miscible organic solvent (such as acetone) was poured into $2.5 \mathrm{~mL}$ of the prepared PS emulsion. For the case of demulsification in acetone (99.5\%, GuangFu Inc) or 1,4-dioxane (99.5\%, Kermel), the PS gel rapidly formed at $25^{\circ} \mathrm{C}$ in several minutes. After the addition of acetonitrile (ACN) (AR, Kermel), the gelation was promoted by a water bath at $25^{\circ} \mathrm{C}$ for $4 \mathrm{~h}$. For gelation after demulsification by $N$-methyl pyrrolidone (NMP) (98\%, Aladdin), N,N-dimethylformamide (DMF) (99.5\%, Kermel), acrylic acid (AA) (AR, Real \& Lead), ethanol (EtOH) (AR, Kermel), or dimethyl sulfoxide (DMSO) (GR, Macklin Inc), the lowest tem- perature at which the gel was obtained is defined as the critical temperature $\left(T_{\mathrm{c}}\right)$. After demulsification by methanol (MeOH) (AR, Kermel), ethylene glycol (EG) (AR, Kermel) and glycerol (TG) (99.5\%, Real \& Lead), the mixtures were treated at 70 and $80^{\circ} \mathrm{C}$, respectively. The gel was then taken out and washed with DI water to remove any residual organic solvent. Finally, the pore structure of the PS monolith could be changed by capillary drying at $70^{\circ} \mathrm{C}$ after solvent exchange with DI water. The gelation mechanism and the structure of the PS monolith were systematically investigated.

\section{Material characterizations}

The microstructure of the samples was observed by transmission electron microscopy (TEM, JEM-2100F) and scanning electron microscopy (SEM, Hitachi S-4800). Dynamic light scattering (DLS, Marvin-Nano ZS) measurements were used to determine the particle size distributions of the as-prepared emulsions as well as the demulsified dispersions. Note that the demulsified samples were measured after ultrasonication for $7 \mathrm{~min}$ to eliminate the unstable aggregation. The monolith bulk densities were measured by a density balance (SartoriousYDK03). Fourier transform infrared spectroscopy (FTIR, Thermo Scientific-Nicolet 380) was performed in the wavenumber range of 400 to $4000 \mathrm{~cm}^{-1}$. Thermogravimetry (TG, TA-Q50) curves were recorded from 30 to $700^{\circ} \mathrm{C}$. Differential scanning calorimetry (DSC, TAQ20) was conducted in the range of $25-200^{\circ} \mathrm{C}$ for the first 2 cycles with heating and cooling rates of $10^{\circ} \mathrm{C} \mathrm{min}^{-1}$. Considering that the first cycle data generally reflects the thermal history of the materials, the data in the second cycle was used for further analysis. Rheological properties of the gels were measured using a RheoPlus MCR301 rheometer with the distance of the two parallel plates fixed at $2 \mathrm{~mm}$ and the oscillation strain at $0.2 \%$. The viscosity of water/organic solvent mixture was also detected by this rheometer, where $15 \mathrm{~mL}$ solution was poured into the container with the detector rotating at the constant speed of $10 \mathrm{rad} \mathrm{s}^{-1}$.

\section{RESULTS AND DISCUSSION}

As shown in Fig. 1, rapid demulsification occurred after the selected water-miscible organic solvent was dropped into the PS microemulsion, and larger clusters or aggregates formed followed by an integrated gel under the appropriate conditions. It was found that the gelation of PS strongly depends on the size of the PS latex particles in the as-polymerized emulsion, the type of organic solvent, and the temperature of the mixture after demulsification. 


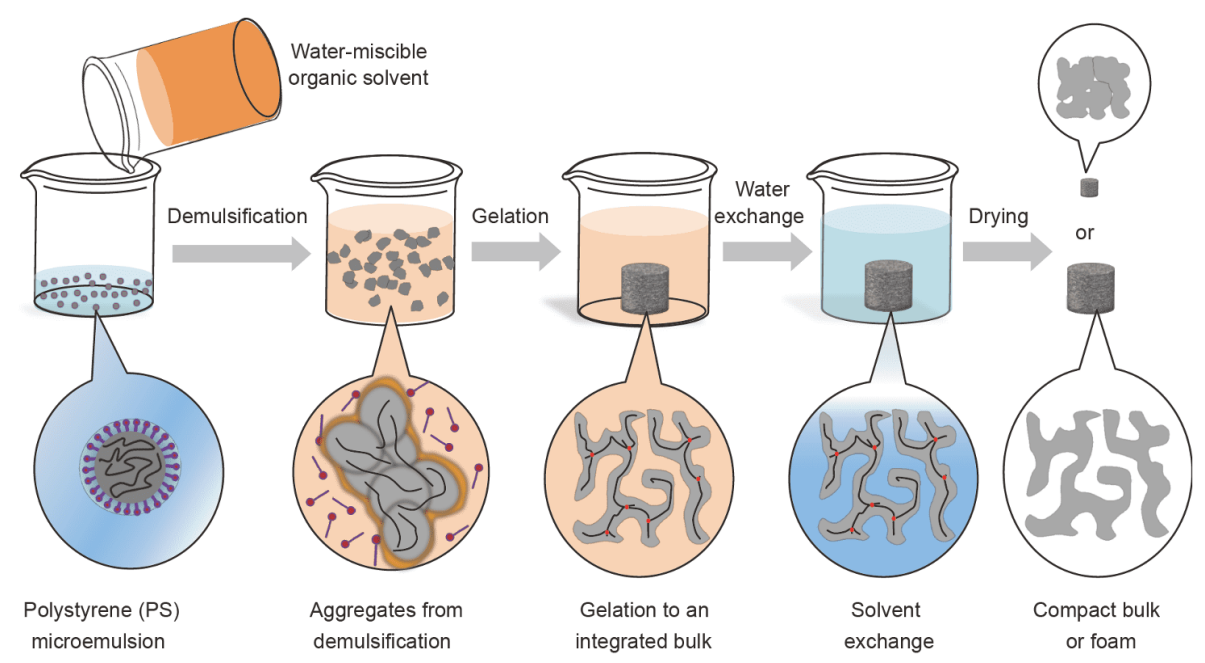

Figure 1 Schematic of the preparation of a PS monolith from a PS microemulsion by adding a water-miscible organic solvent for demulsification and followed by gelation and controlled drying.

Moreover, the pore structure of the monolith could be tuned by evaporation-induced capillary drying after solvent exchange with water. That is, the PS gel could be dried to produce either a compact PS bulk or a porous PS aerogel by controlled densification.

The influence of the size of the primary latex particles on PS gelation was first studied. Considering the difficulty of observing this step in the rapid gelation with acetone, $\mathrm{EtOH}$ was used as the demusification solvent. A series of PS emulsions denoted samples 1-5 with increasing latex particle size was prepared (see Table S1 for detailed synthesis recipes). DLS studies show that the average latex particle sizes for samples 1-5 are 42, 45, 50, 70, and $470 \mathrm{~nm}$, respectively (Fig. 2a and Fig. S1a), which are slightly higher than those observed in TEM images (Fig. S2), due to the solvent effect and the adsorbed surfactant layer [19]. When EtOH was dropped into the PS emulsion, the average sizes of the PS particles in these five solutions respectively increased to around 400, 275, 186, 106, and $560 \mathrm{~nm}$ (Fig. 2a and Fig. S1b). It should be mentioned that after demulsification, the three PS emulsions with the smaller particles showed a marked shift in size distribution compared with the two counterparts with larger particles, which might be attributed to more coalescence along with stronger Brownian motion as well as a structural change driven by a tendency to reduce specific surface energy [20]. Another factor may be due to the higher mobility of PS chains on the surface than in the bulk $[21,22]$. Therefore, the smaller the particle size, the higher the surface area, and the more likely the inter-particle interaction of the polymer chains, leading to a tight aggregation that is hard to break by ultrasonication before the DLS measurement. This additional interaction is partially ascribed to the $\pi-\pi$ bonding between aromatic groups [23], but is mostly the result of the entanglement of the polymer chains, which is a kind of physical cross-linking [24,25]. This promotes the formation of a three-dimensional integrated material at an appropriate temperature $\left(80^{\circ} \mathrm{C}\right.$ for $\left.\mathrm{EtOH}\right)$ as illustrated by the first three samples in Fig. 2b. In contrast, the two emulsions with larger primary particle sizes failed to form a PS gel due to insufficient aggregation and a lack of chain entanglement between the primary particles. Similar observations are presented in Fig. S3a when acetone was used as the demulsifier for rapid gelation. In rheological testing, the absence of a modulus change with increasing frequency implies the outstanding stability of the asformed gel structure (Fig. S3b).

Gelation is highly dependent on the organic solvent and its volume. The solution with $80 \mathrm{vol} . \%$ of a water-miscible organic demulsifier showed better gelation than those with a lower volume fraction (Fig. S4), indicating that a larger volume of demulsifier favours gelation. This is attributed to the formation of more crosslinks accompanied by more intense desolvation. In the following studies, the amount of water-miscible organic solvent was fixed at $80 \mathrm{vol} . \%$. The type of solvent for demulsification is also a key factor in determining the PS gelation. As shown in Fig. 2c, the gelation ability of various solvents can be divided into three types: (i) a bulk gel is directly formed at room temperature for acetone, 1,4-dioxane and ACN; (ii) heating is necessary to obtain an integrated monolith for DMF, NMP, EtOH, AA, and DMSO; (iii) no obvious changes occur even at a high temperature for $\mathrm{MeOH}$, EG 

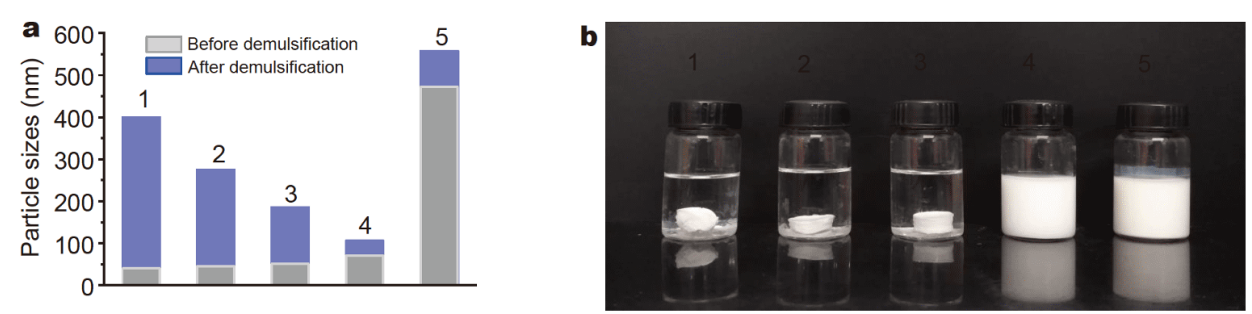

c

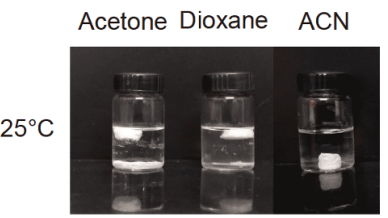

DMF NMP EtOH AA DMSO
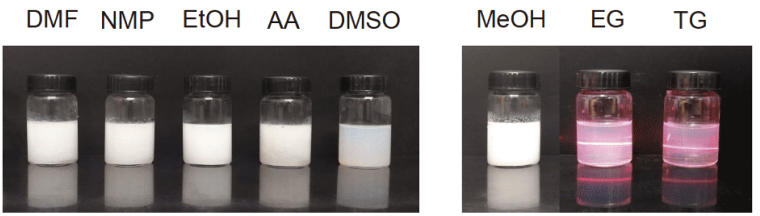

After heating
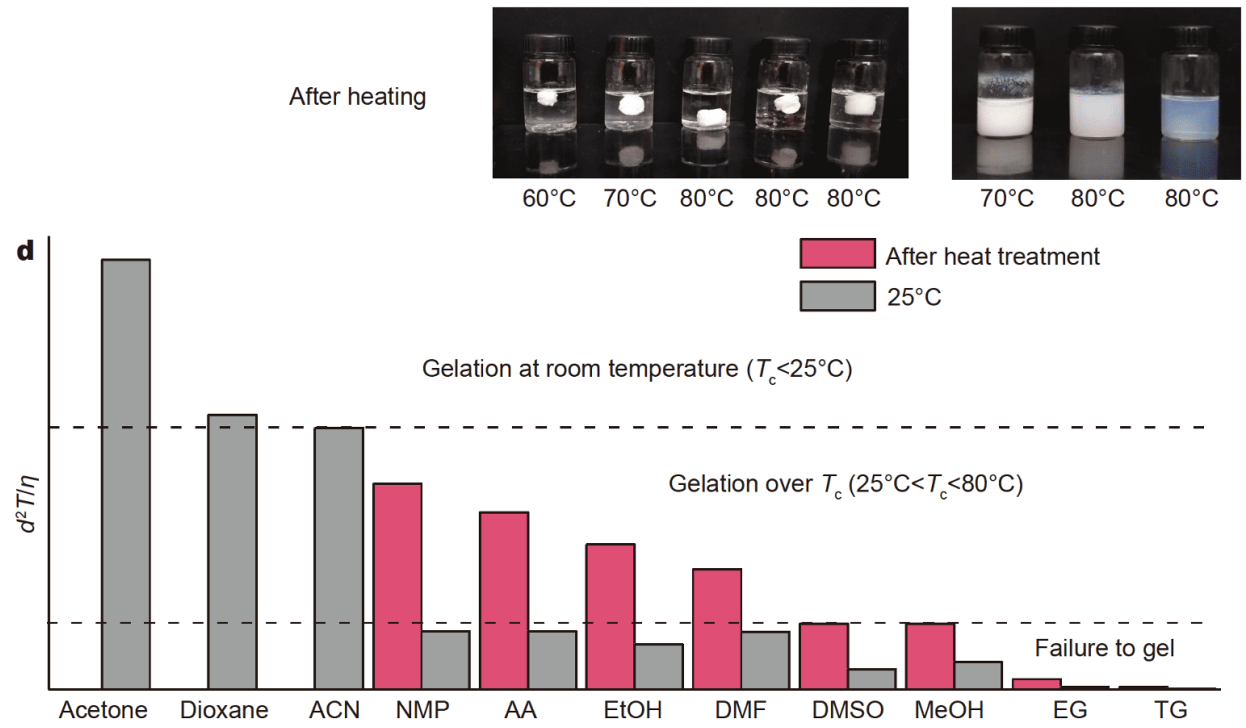

Figure 2 (a) Comparison of the average particle sizes of the PS emulsions before and after demulsification by EtOH. (b) Optical photograph of the PS emulsions with different particle sizes after demulsification by $\mathrm{EtOH}$ and subsequent gelation at $80^{\circ} \mathrm{C}$ for $3 \mathrm{~h}$. (c) Optical photographs of the demulsification at $25^{\circ} \mathrm{C}$ and subsequent gelation at the lowest gelation temperature $\left(T_{\mathrm{c}}\right)$ or boiling point of the solvent mixture. (d) $d^{2} T / \eta$ value under either ambient temperature or $T_{\mathrm{c}}$ for all the chosen organic solvents, showing that the gelation ability of various solvents can be divided into three types: gelation under $25^{\circ} \mathrm{C}$, gelation at $25-80^{\circ} \mathrm{C}$, and failure to gel above $80^{\circ} \mathrm{C}$ or the boiling point of solvent mixtures.

and TG. It is evident that the solvent largely determines the size of secondary colloidal particle $(d)$. DLS results showed that particle sizes increase significantly in acetone, ACN, 1,4-dioxane and NMP, while the particle size changes are relatively small in $\mathrm{MeOH}, \mathrm{EG}$ and TG (Fig. S5). The colloidal nature of the dispersions confirmed by the Tyndall effect also suggested the limited development of secondary particles in EG and TG. More specifically, the size change of the colloidal particles and the gelation phenomena are closely associated with the three-dimensional Hansen solubility parameter (HSP, $\delta_{\mathrm{t}}$ ) of the solvents, which is commonly used to evaluate the miscibility of two species with each other. PS $\left(\delta_{\mathrm{t}} \approx 19.8\right)$ dispersed in a solvent with a similar $\delta_{\mathrm{t}}$ value results in lower interfacial tension and larger secondary particles
[26]. In addition, solvents with a similar $\delta_{\mathrm{t}}$ value cause the PS latex to swell significantly and make the PS polymer chains more flexible [27], so there would be increased free space and more mobility for significant rearrangement and crosslinking of the polymer chains in each particle. Details of the three-dimensional HSP values of PS and the organic solvents, particle sizes and corresponding phenomena after adding the solvent are listed in Table S2 and the $d$ value is plotted against $\delta_{\mathrm{t}}$ for the solvent in Fig. S6. We found that $d$ generally decreased with increasing $\delta_{\mathrm{t}}$, and a larger secondary particle implies more inter-particle crosslinking and thus easier gelling.

The effect of organic solvents on the gelation ability is not only related to the $\delta_{\mathrm{t}}$ value but also to the diffusion coefficient $(D)$ of the secondary colloidal particles. $D$ is a 
crucial parameter reflecting the speed of the random thermal movement of the particles, i.e. the possibility of collision and fusion of the secondary particles or chains before they entangle into a bulk gel. Taking type (ii) gel formation as an example, the increased $D$ value of the secondary colloidal particles at high temperature is beneficial for gelation. According to the Einstein-Stocks equation (Equation (S1)), $D$ increases with temperature and decreases with viscosity $\eta$ and particle size. That is, $D$ is proportional to $T / d \eta$ (the $\eta$ values for various water/ organic solvent mixtures are listed in Table S3), so that increasing the temperature leads to an increased diffusion rate, gives rise to stronger collisions and physical crosslinking between adjacent secondary particles, and macroscopic gelling is promoted. In other words, the gelation ability is determined by both diffusion of the particles $(D$ value) and the $\delta_{\mathrm{t}}$ value of the solvent which strongly influences the secondary particle mass as mentioned above, and correlates with the cube of the diameter $\left(d^{3}\right)$ of the secondary particles given by Equation (S2). Therefore, the parameter $d^{3} \times T / d \eta=d^{2} T / \eta$ can be used to predict the gelation ability of various organic solvents and quickly determine the suitable gelation condition. As shown in Fig. $2 \mathrm{~d}$, the $d^{2} T / \eta$ values at room temperature fall into three categories, which is basically consistent with the classification in Fig. 2c. For example, it can be used to explain the special cases of NMP and AA, which cannot form a PS gel at room temperature even their HSP values are closer to PS than that to ACN. This is due to the lower $d^{2} T / \eta$ value at room temperature as a result of the high viscosities of NMP and AA. In order to promote gel formation, a higher temperature is necessary to increase the $d^{2} T / \eta$ value, which also means a lower viscosity of solvent and thus favours the movement of secondary particles and increases the amount of entanglement of the polymer chains. As a result, we define $T_{\mathrm{c}}$ as the lowest temperature at which a PS gel is formed. It is found that $T_{\mathrm{c}}$ for NMP is $70^{\circ} \mathrm{C}$ and a higher temperature speeds up PS gelation.

To further understand the gel formation mechanism, the thermal properties and chemical compositions of the samples before and after gelation were investigated using DSC and FTIR. The PS monolith formed at $70^{\circ} \mathrm{C}$ (PS-M) and PS powder obtained by filtration of the same suspension demulsified by DMF (PS-P) were collected. As shown in the DSC curves in Fig. 3a and Fig. S7, PS-M has a much higher transition temperature $\left(\sim 108^{\circ} \mathrm{C}\right)$ than PS$\mathrm{P}\left(\sim 89^{\circ} \mathrm{C}\right)$, which confirms the limited mobility of PS chains in the monolith due to the much larger amount of crosslinking after gelation. In addition, it was found that the demulsification induced by organic solvents is totally different from that caused by inorganic salt solutions. In both the PS-M and PS-P samples, no surfactants remained after the addition of DMF which is shown by the absence of a $-\mathrm{SO}_{3}{ }^{-}$signal from SDS species in the FTIR spectra and a slight weight loss below $350^{\circ} \mathrm{C}$ in the thermogravimetric (TG) curves (Fig. $3 \mathrm{~b}$ and Fig. S8a) [28]. This implies that the surfactant species were entirely desorbed from the PS latex and dissolved in the liquid after the addition of the organic solvent, which led to an increased affinity between the PS particles and greatly facilitated their collision and fusion. This process is quite unlike the previously reported latex swelling process for reactive gelation. In contrast, there is still obvious residual SDS surfactant for demulsified samples induced by an excess $\mathrm{MgCl}_{2}$ solution (PS- $\mathrm{MgCl}_{2}$ ) even after an extra rinse, where the particle coagulation is caused by the charge neutralization. Thus, it is difficult to form a gel under the same conditions because of the poor interparticle affinity caused by the existence of a surfactant layer [29]. Interestingly, the $\mathrm{MgCl}_{2}$-induced precipitation still has gelation ability which is seen once the organic
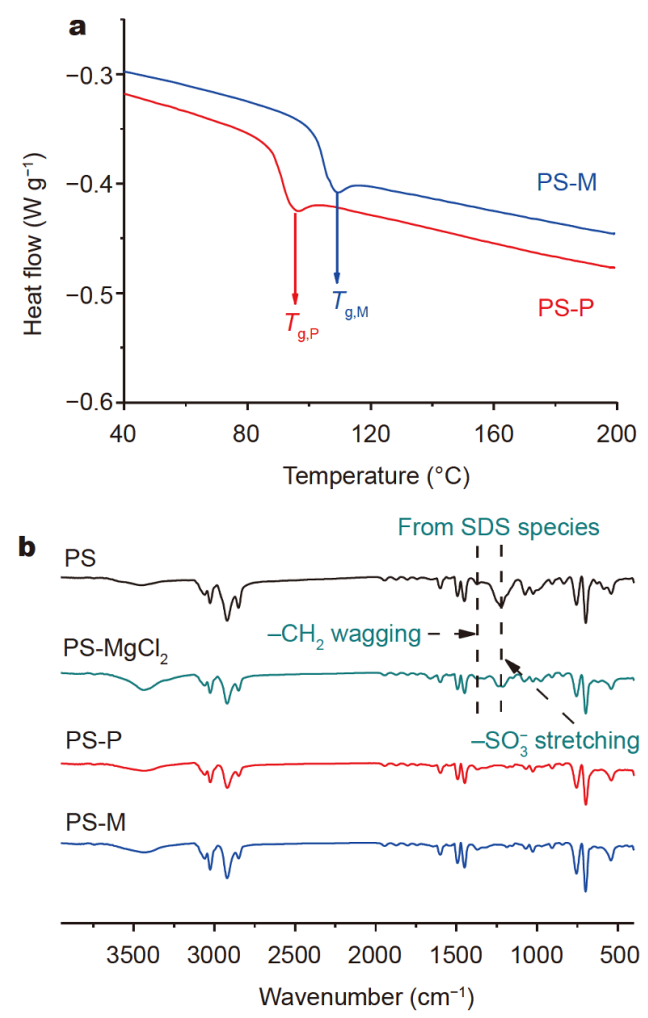

Figure 3 (a) DSC curves of a PS monolith (PS-M) and PS powder (PS$\mathrm{P})$ collected from the same microemulsion after DMF demulsification. (b) FTIR spectra of PS-M, PS- $\mathrm{MgCl}_{2}$, PS-P and PS particles obtained by direct filtration of the PS microemulsion. 
solvent is introduced (Fig. S8b). The above result suggests that the complete desorption of SDS surfactant induced by the organic solvent demulsification plays a key role in PS gel formation, which is also important in avoiding any surfactant residue in the PS monoliths.

The pore structure of the as-formed PS gels can be further tailored by capillary drying, which has already proved useful for shrinking the interconnected flexible graphene network as reported in a previous paper [17]. Here, the trapped solvent in the gels was first exchanged with water, which has a high surface tension of $72.7 \mathrm{mN} \mathrm{m}^{-1}$, and the gels were then dried at $70^{\circ} \mathrm{C}$. The PS monoliths obtained showed quite different degrees of shrinkage for gels prepared using different solvents. As shown in Fig. 4a, PS foams were only obtained for the gels prepared using acetone, $\mathrm{EtOH}$ and $\mathrm{ACN}$, while both porous and dense structures were obtained for the remainder after solvent removal. As observed from the SEM images, PS foams have a porous structure constructed by relatively uniform-sized particles (Fig. S9a-c), whose size was consistent with the size of the PS aggregations after solvent demulsification, while the very stiff monolith has a large volume shrinkage and a compact microstructure (Fig. S9d). To achieve controlled densification, both the gelation time and degree of solvent exchange must be taken into consideration. Here, DMSO was used as the demulsifying solvent to study the impact of these two factors on the final structure of the PS monolith, since this system has a long gelation time. The improved storage modulus $\left(G^{\prime}\right)$, loss modulus $\left(G^{\prime \prime}\right)$ and shear viscosity in rheological testing, as well as a reduced gel volume, indicating the increased mechanical strength and degree of entanglement of the PS gel as the gelation time was prolonged from 5 to $10 \mathrm{~h}$ (Fig. $4 \mathrm{~b}$ and Fig. S10) [30]. As shown in Fig. 4c, the densities of the PS monoliths could be changed from 0.06 to $1.14 \mathrm{~g} \mathrm{~cm}^{-3}$ and gradually decreased with the gelation time, while an additional solvent exchange with water did not help in achieving higher densities. For example, almost no shrinkage during drying could be observed after a second water exchange when the gelation time was longer than $8.5 \mathrm{~h}$. These monoliths thus had much lower densities (less than $0.086 \mathrm{~g} \mathrm{~cm}^{-3}$ ). Considering the above results, it is likely that increasing the gelation time and the degree of water exchange of the PS gel is detrimental to capillary shrinkage of the PS network. TG analysis was used to evaluate the amount of residual organic solvent, which is a direct indicator of the degree of solvent exchange in the PS gel. PS monoliths prepared with a shorter gelation time and less water exchange (samples of $5 \mathrm{~h}-1^{\text {st }}, 5 \mathrm{~h}-2^{\text {nd }}$ and $10 \mathrm{~h}-1^{\text {st }}$ ) showed much higher weight losses in the temperature range of $200-350^{\circ} \mathrm{C}$ (Fig. $4 \mathrm{~d}$ ), which indicates that some residual DMSO is difficult to evaporate during capillary drying and is thus trapped in channels in
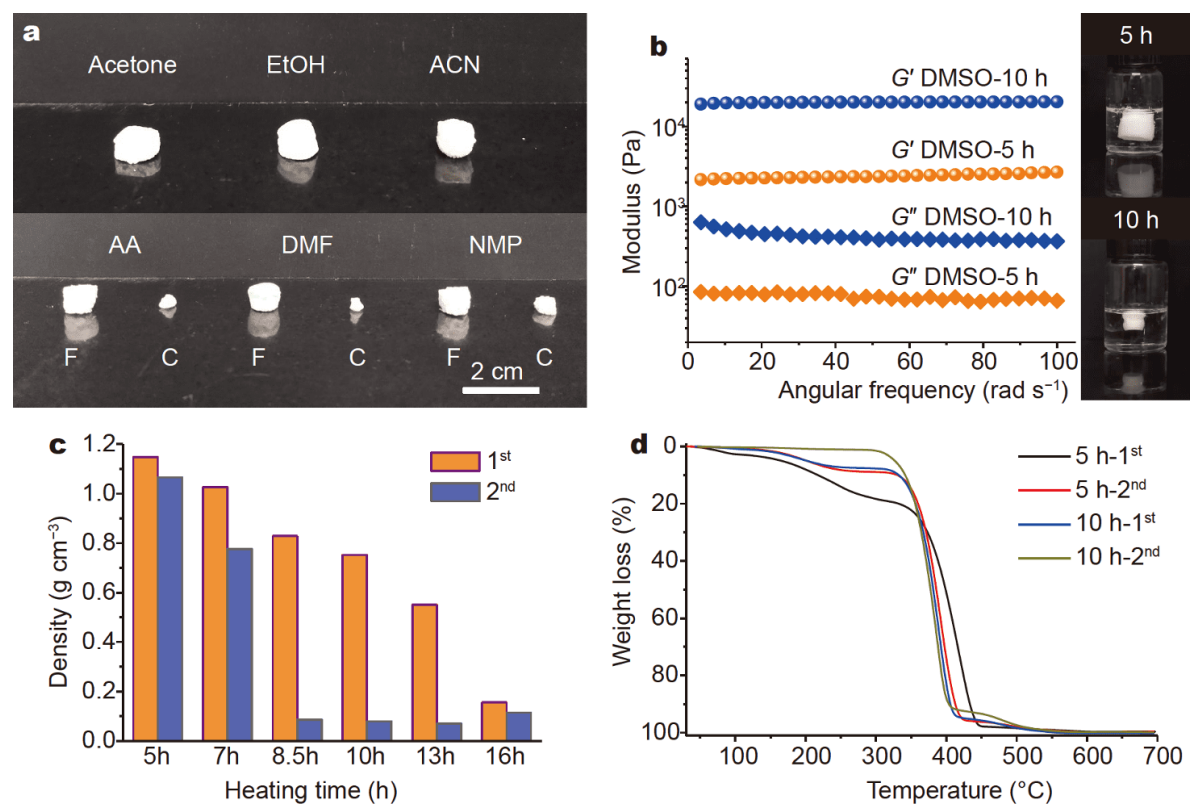

Figure 4 (a) Optical photograph of PS monoliths with the two extreme structures of a porous foam (F) and a compact form (C) prepared using different solvents. (b) The rheological storage modulus $\left(G^{\prime}\right)$ and loss modulus $\left(G^{\prime \prime}\right)$ as a function of oscillation frequency for the PS gels with different gelation times in DMSO. (c) Dependence of density on the gelation time of a PS gel when the trapped solvent is exchanged with water once $\left(1^{\text {st }}\right)$ or twice $\left(2^{\text {nd }}\right)$. (d) TG curves of PS monoliths with different gelation times and degrees of water exchange. 
the monolith. These three samples all had high densities due to the effective shrinkage and were characterized by a compact microstructure as observed in the SEM images (Fig. S11). In contrast, the ultralight $10 \mathrm{~h}-2^{\text {nd }}$ sample has a highly porous structure with abundant macropores, which corresponded to negligible residual DMSO as confirmed by the TG analysis. These results suggest that shortening the gelation time and reducing the water exchange time cause more residual DMSO and a higher density. This is largely because the gel with less chain entanglement absorbs more DMSO to make the PS skeleton flexible, which permits easier shrinkage of the PS network during capillary drying. Moreover, a certain amount of residual organic solvent in the PS gel with less water exchange retains the stretching ability of the PS chains and thus also helps realize capillary shrinkage during subsequent removal of the trapped solvent. That is to say, the degree of shrinkage of the PS gel is associated with the state of the PS skeleton. The PS gel with a less entangled chain structure and more absorbed organic solvent is more readily shrunk during capillary drying. Consequently, the pore structure of the PS monoliths can be finely tuned by varying the above factors. A combination of partial capillary-drying and subsequent freezedrying to remove the trapped water in the assembled gels can also be used to achieve the structure control of the PS monoliths. As shown in Fig. S12, an increase of the capillary-drying time leads to larger degree of shrinkage and significant changes in the microstructure as the loose porous structure changes to a more compact microstructure.

\section{CONCLUSIONS}

In summary, a new strategy for the gelation of PS latex from a microemulsion with an emphasis on the gelation mechanism was proposed. A stable PS gel is obtained by the gelation of the PS latex after demulsification initiated by a selected water-miscible organic solvent, which, to the best of our knowledge, is the first report on PS gelation resulting from a demulsification-induced chain entanglement. It was found that the gelation ability of the organic solvents can be evaluated by the $d^{2} T / \eta$ value, which takes into account the influence of both the solvent and the diffusion ability of the aggregates. In addition, the strategy proposed here has a great advantage in the structure control of PS monoliths, which can be easily regulated by controlling the gelation time, degree of water exchange of the gel, and the capillary-drying time, etc. This new gelling strategy avoids the use of shaping equipment or templates as well as the addition of a crosslinking agent. It eliminates the needs of a porogenic solvent or a large amount (5 wt.\%-50 wt.\%) of surfaceactive material for stabilization. It is believed that this simple alternative PS gelation method provides a promising way to produce functional polymer monoliths and may be extended to a wide range of other polymers for bulk material production.

Received 22 October 2020; accepted 1 February 2021; published online 9 April 2021

1 Feinle A, Elsaesser MS, Hüsing N. Sol-gel synthesis of monolithic materials with hierarchical porosity. Chem Soc Rev, 2016, 45: 3377-3399

2 Röttger $\mathrm{M}$, Domenech $\mathrm{T}$, van der Weegen $\mathrm{R}$, et al. Highperformance vitrimers from commodity thermoplastics through dioxaborolane metathesis. Science, 2017, 356: 62-65

3 Nutz FA, Ruckdeschel P, Retsch M. Polystyrene colloidal crystals: Interface controlled thermal conductivity in an open-porous mesoparticle superstructure. J Colloid Interface Sci, 2015, 457: 96-101

4 de Assis GC, Skovroinski E, Leite VD, et al. Conversion of "waste plastic" into photocatalytic nanofoams for environmental remediation. ACS Appl Mater Interfaces, 2018, 10: 8077-8085

5 Ye Y, Jin M, Wan D. One-pot synthesis of porous monolithsupported gold nanoparticles as an effective recyclable catalyst. J Mater Chem A, 2015, 3: 13519-13525

6 Chen Q, Shi T, Han F, et al. Porous polystyrene monoliths and microparticles prepared from core cross-linked star (CCS) polymers-stabilized emulsions. Sci Rep, 2017, 7: 8493

7 Pang B, Liu H, Liu P, et al. Water-in-oil Pickering emulsions stabilized by stearoylated microcrystalline cellulose. J Colloid Interface Sci, 2018, 513: 629-637

8 Chen C, Li R, Xu L, et al. Three-dimensional superhydrophobic porous hybrid monoliths for effective removal of oil droplets from the surface of water. RSC Adv, 2014, 4: 17393-17400

9 Silverstein MS. PolyHIPES: Recent advances in emulsiontemplated porous polymers. Prog Polym Sci, 2014, 39: 199-234

10 Sun G, Li Z, Ngai T. Inversion of particle-stabilized emulsions to form high-internal-phase emulsions. Angew Chem Int Ed, 2010, 49: 2163-2166

11 Menner A, Ikem V, Salgueiro M, et al. High internal phase emulsion templates solely stabilised by functionalised titania nanoparticles. Chem Commun, 2007, 41): 4274-4276

12 Marti N, Quattrini F, Butté A, et al. Production of polymeric materials with controlled pore structure: the "reactive gelation" process. Macromol Mater Eng, 2005, 290: 221-229

13 Lamprou A, Köse I1, Storti G, et al. Synthesis of macroporous polymer particles using reactive gelation under shear. Langmuir, 2014, 30: 6946-6953

14 Wang G, Yu B, Chen S, et al. Template-free synthesis of polystyrene monoliths for the removal of oil-in-water emulsion. Sci Rep, 2017, 7: 6534

15 Kim SJ, Raut P, Jana SC, et al. Electrostatically active polymer hybrid aerogels for airborne nanoparticle filtration. ACS Appl Mater Interfaces, 2017, 9: 6401-6410

16 Schüler F, Schamel D, Salonen A, et al. Synthesis of macroporous polystyrene by the polymerization of foamed emulsions. Angew Chem Int Ed, 2012, 51: 2213-2217

17 Tao Y, Xie X, Lv W, et al. Towards ultrahigh volumetric capaci- 
tance: graphene derived highly dense but porous carbons for supercapacitors. Sci Rep, 2013, 3: 2975

18 Li P, Li H, Han D, et al. Packing activated carbons into dense graphene network by capillarity for high volumetric performance supercapacitors. Adv Sci, 2019, 6: 1802355

19 Khan MF, Singh MK, Sen S. Measuring size, size distribution, and polydispersity of water-in-oil microemulsion droplets using fluorescence correlation spectroscopy: Comparison to dynamic light scattering. J Phys Chem B, 2016, 120: 1008-1020

20 Sun $\mathrm{H}$, Jiao $\mathrm{R}, \mathrm{Xu} \mathrm{H}$, et al. The influence of particle size and concentration combined with $\mathrm{pH}$ on coagulation mechanisms. J Environ Sci, 2019, 82: 39-46

21 Ellison CJ, Torkelson JM. The distribution of glass-transition temperatures in nanoscopically confined glass formers. Nat Mater, 2003, 2: 695-700

22 Kim H, Cang Y, Kang E, et al. Direct observation of polymer surface mobility via nanoparticle vibrations. Nat Commun, 2018, 9: 2918

23 Huo H, Li K, Wang Q, et al. Self-assembly and optical property of triblock copolymers made of polystyrene and oligo( $p$-phenyleneethynylene) in different mixtures of toluene and hexane. Macromolecules, 2007, 40: 6692-6698

24 Busse WF. The physical structure of elastic colloids. J Phys Chem, 1932, 36: 2862-2879

25 Flory PJ. Network structure and the elastic properties of vulcanized rubber.. Chem Rev, 1944, 35: 51-75

26 Liu B, Zhang $\mathrm{M}$, Cheng $\mathrm{H}$, et al. Large-scale and narrow dispersed latex formation in batch emulsion polymerization of styrene in methanol-water solution. Colloid Polym Sci, 2014, 292: 519-525

27 Li B, Zhou J, Xu X, et al. Solvent quality affects chain conformational order at the polymer surface revealed by sum frequency generation vibrational spectroscopy. Polymer, 2013, 54: 1853-1859

28 Banjare MK, Kurrey R, Yadav T, et al. A comparative study on the effect of imidazolium-based ionic liquid on self-aggregation of cationic, anionic and nonionic surfactants studied by surface tension, conductivity, fluorescence and FTIR spectroscopy. J Mol Liquids, 2017, 241: 622-632

29 Feng S, Li ZY, Liu R, et al. Glass transition of polystyrene nanospheres under different confined environments in aqueous dispersions. Soft Matter, 2013, 9: 4614

30 Zhang X, Yang H, Song Y, et al. Rheological behaviors of randomly crosslinked low density polyethylene and its gel network. Polymer, 2012, 53: 3035-3042

Acknowledgements This work was financially supported by the National Science Fund for Distinguished Young Scholars of China (51525204), and the National Natural Science Foundation of China (51702229).

Author contributions Yang QH, Tao Y and Li D conceived and designed the experiments. Li D and Xia J conducted the material synthesis and characterization. Li D and Tao Y took charge of the data collection and analysis. Wu Z and Han J performed the SEM and TEM experiments. Deng Y, Li P and Shang T helped with the data analysis. Li $\mathrm{D}$ and Tao $\mathrm{Y}$ wrote the paper with further inputs from other authors. All the authors discussed the results and contributed to the manuscript.

Conflict of interest The authors declare that they have no conflict of interest.
Supplementary information Experimental details and supporting data are available in the online version of the paper.

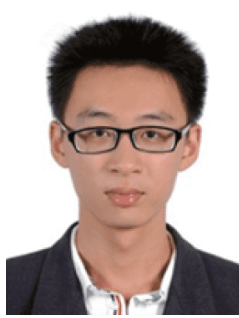

Dewang Li received his Bachelor's degree and Master's degree in materials science and engineering from Tianjin University in 2013 and 2016, respectively, and currently studies as a $\mathrm{PhD}$ candidate under the guidance of Prof. QuanHong Yang. His research focuses on the gelation and liquid phase assembly of functional materials and their applications in energy storage.

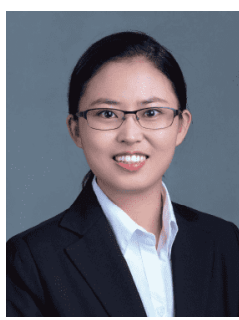

Ying Tao is now an associate professor at the School of Chemical Engineering and Technology, Tianjin University. Her current research interests focus on the assembly of low-dimensional materials, carbon-based materials and their applications in electrochemical energy storage devices and environmental remediation.

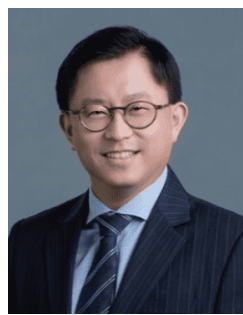

Quan-Hong Yang joined Tianjin University as a full professor in 2006 and became a Chair Professor in the same university in 2016. His research focuses on novel functional carbon materials with the applications in energy and environmental issues. Specifically, he has made significant advances in high volumetric performance EES devices and the catalysis in lithiumsulfur batteries. See http://nanoyang.tju.edu.cn for more details about Nanoyang Group.

\section{聚苯乙烯微乳液的凝胶化及其块体的快速制备和 结构调控}

李德望 ${ }^{1}$, 邓亚茜 ${ }^{2}$, 夏静怡 $^{1}$, 吴志坦 ${ }^{1}$, 尚童秷 ${ }^{1}$, 李培 ${ }^{1}$, 韩俊伟 ${ }^{1}$, 陶莹 ${ }^{1^{*}}$, 杨全红 ${ }^{1,3}$

摘要 本文利用一种新的聚苯乙烯(PS)微乳液的凝胶化现象, 制备 了结构稳定的PS凝胶, 并通过可控的毛细收缩过程获得了具有不 同孔隙结构的PS块体. 对PS微乳液的凝胶化机制的系统研究表明, 在PS微乳液中加入适量特定的水溶性有机溶剂后, 可实现PS微乳 液的破乳，PS乳胶粒子周围的表面活性剂完全解吸附, 并自发团聚 成二次颗粒, 在适当温度条件下可通过分子链缠结组装形成三维 多孔凝胶. 不同溶剂实现PS微乳液凝胶化的能力可以通过 $d^{2} T / \eta$ 参 数的大小衡量和评估. 通过进一步调整成胶时间与溶剂置换程度, PS 凝胶可在后续的干燥过程中实现不同程度的收缩, 从而得到密 度范围在 $0.06-1.14 \mathrm{~g} \mathrm{~cm}^{-3}$ 的多孔或致密PS块体. 这种PS凝胶化策 略是一种简单高效的PS成型方法, 避免了成型设备、模板、成孔 剂以及大量表面活性剂的应用, 有望成为制备功能高分子聚合物 块体材料的新方法. 